\title{
Exploring the conformal window: SU(2) gauge theory on the lattice
}

\section{Tuomas Karavirta*}

Department of Physics, P.O.Box 35 (YFL),

FI-40014 University of Jyväskylä, Finland,

and

Helsinki Institute of Physics, P.O. Box 64,

FI-00014 University of Helsinki, Finland

E-mail: tuomas.karavirta@jyu.fi

\section{Jarno Rantaharju}

Department of Physics and Helsinki Institute of Physics, P.O.Box 64, FI-00014 University of Helsinki, Finland

Email: jarno.rantaharju@helsinki.fi

\section{Kari Rummukainen}

Department of Physics and Helsinki Institute of Physics, P.O.Box 64, FI-00014 University of Helsinki, Finland

Email: kari.rummukainen@helsinki.fi

\section{Kimmo Tuominen}

Department of Physics, P.O.Box 35 (YFL),

FI-40014 University of Jyväskylä, Finland,

and

Helsinki Institute of Physics, P.O. Box 64,

FI-00014 University of Helsinki, Finland

Email: kimmo.i.tuominenajyu.fi

We study the SU(2) gauge theory on the lattice with different numbers of fermions in the fundamental representation of the gauge group to explore the gauge theory phase diagram. We find evidence for an infrared fixed point for ten flavors. The theory with six flavors shows behaviors compatible with the existence of a (quasi) stable fixed point, but the large errors in the present data do not allow for decisive confirmation of this.

The XXIX International Symposium on Lattice Field Theory, Lattice2011

July , 2011

\footnotetext{
* Speaker.
} 


\section{Introduction}

There exists a class of gauge theories where, under the renormalization group evolution, the coupling shows asymptotic freedom at small distances, analogously to QCD, but flows to a fixed point at large distances where the theory hence looks conformal. Such theories have applications in beyond Standard Model model building. These include unparticles, i.e. an infrared conformal sector coupled weakly to the Standard Model [1], and (extended) technicolor scenarios, that explain the masses of the Standard Model gauge bosons and fermions via strong coupling gauge theory dynamics [2, 3, 4]. In addition to direct applications to particle phenomenology, the phase diagrams of gauge theories, as a function of the number of colours, $N$, flavours $N_{f}$ and fermion representations, are interesting from the purely theoretical viewpoint of understanding the nonperturbative gauge theory dynamics from first principles. While several semianalyitc methods to estimate the vacuum phase diagram of a gauge theory exist, the only truly first principle method is constituted by lattice simulations. Several initial studies have appeared in literature: for example SU(2) with fundamental representation fermions [5], SU(2) with adjoint fermions [6, 7, 8, 9, 10, 11] and SU(3) with fermions in the fundamental $[12,13,14]$ or in the two-index symmetric [15], i.e. the sextet, representation.

The studies with Wilson fermions are subject to lattice artifacts proportional to the lattice spacing $a$, and a program to cancel these lattice artifacts has been devised [16, 17]. As a motivation for this improvement, consider the measurement of the running coupling using the Schrödinger functional method: The coupling is measured using a background field and the scale is set by the finite size of the lattice. We consider a lattice of volume $V=L^{4}=(\mathrm{Na})^{4}$. The spatial links at the $t=0$ and $t=L$ boundaries are fixed to constant values, while the spatial boundary conditions are periodic. The fermion fields are set to vanish at the $t=0$ and $t=L$ boundaries and have twisted periodic boundary conditions in spatial directions: $\psi(x+L \hat{i})=\exp (i \pi / 5) \psi(x)$. At the classical level, the boundary conditions generate a constant chromoelectric field and the derivative of the action with respect to $\eta$ can be easily calculated:

$$
\frac{\partial S^{\mathrm{cl}}}{\partial \eta}=\frac{k}{g_{0}^{2}},
$$

where $k$ is a function of $N=L / a$ and $\eta$.At the full quantum level the coupling is defined by

$$
\left\langle\frac{\partial S}{\partial \eta}\right\rangle=\frac{k}{g^{2}} .
$$

The perturbative step scaling function defined using the evolution of the renormalized coupling $g$ from scale $L$ to scale $s L$, i.e.

$$
\begin{aligned}
\Sigma(u, s, L / a) & =\left.g^{2}\left(g_{0}, s L / a\right)\right|_{g^{2}\left(g_{0}, L / a\right)=u} \\
& =u+\left(\Sigma_{1,0}+\Sigma_{1,1} N_{f}\right) u^{2} .
\end{aligned}
$$

The second line gives the formula in perturbation theory to one loop order, and the fermion contribution is denoted by $\Sigma_{1,1}$. To evaluate these perturbative contributions we use the methods in $[18,19]$, and choose $s=2$. The continuum limit of $\Sigma_{1,1}$ is given by the fermionic contribution to 


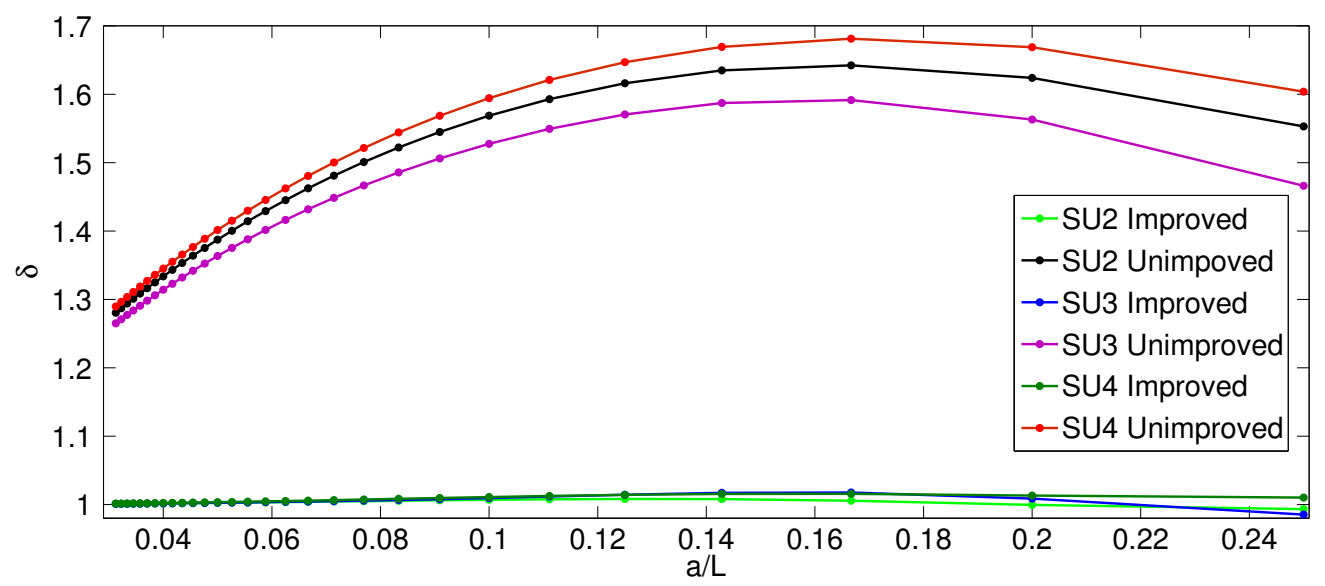

Figure 1: Contribution of a massless Wilson quark to the step scaling function normalized to its continuum value at one loop order in perturbation theory. The top three curves show the result for gauge groups SU(4), SU(3) and SU(2) (from top to bottom) for unimproved Wilson fermions, while the lower three curves show the result after $\mathscr{O}(a)$ improvement has been taken into account.

the one loop coefficient $b_{0}=\beta_{0} /\left(16 \pi^{2}\right)$ of the beta function, i.e.

$$
\delta=\lim _{L / a \rightarrow 0} \Sigma_{1,1} /\left(2 N_{f} b_{0,1} \ln 2\right),
$$

where $b_{0,1}=1 /\left(24 \pi^{2}\right)$.

The results for the one loop fermion contribution is shown in figure 1 both for unimproved Wilson fermions and with $\mathscr{O}(a)$ improvement. One immediately observes that without improvement, $\Sigma_{1,1}$ depends strongly on $L / a$ and approaches the continuum limit only for large lattices, while with improvement the large lattice artefacts are absent. Clearly this motivates the need to use improved actions in the lattice studies of these theories with Wilson fermions.

In our nonperturbative study we use Symanzik improved Wilson fermions to remedy the discretization errors. We measure the running coupling in both models and the mass anomalous dimension in the model with six fundamental fermions.

\section{The model and theoretical tools}

In this section we will introduce the model and some theoretical tools regarding the step scaling function and anomalous dimension of the mass operator. We use the basic Wilson Lattice action

$$
S_{0}=S_{G}+S_{F},
$$

where $S_{G}$ is the standard Wilson plaquette action and and $S_{F}$ is the clover improved Wilson action

$$
S_{F}=a^{4} \sum_{\alpha=1}^{N_{f}} \sum_{x}\left[\bar{\psi}_{\alpha}(x)\left(i D+m_{0}\right) \psi_{\alpha}(x)+a c_{\mathrm{sw}} \bar{\psi}_{\alpha}(x) \frac{i}{4} \sigma_{\mu v} F_{\mu v}(x) \psi_{\alpha}(x)\right]
$$

where $D$ is the standard Wilson-Dirac derivative operator including the doubler term. We set the improvement coefficient $c_{\mathrm{sw}}$ to the perturbative value [20] $c_{\mathrm{sw}}=1+0.1551(1) g_{0}^{2}+O\left(g_{0}^{4}\right)$. We have 
performed a few short measurements that imply that at strong coupling this is close to the correct nonperturbative value for $c_{\mathrm{sw}}$ with 6 and 10 fermion flavours. We also include the perturbative improvement at the Schrödinger functional boundaries as described in [21].

There is a relation between step scaling funtion (1.3) and the $\beta$ function:

$$
-2 \ln (s)=\int_{u}^{\sigma(u, s)} \frac{d x}{\sqrt{x} \beta(\sqrt{x})} .
$$

Near the fixed point $\beta$-function is small and (2.3) can be approximated with

$$
\beta(g) \approx \frac{g}{2 \ln (2)}\left(1-\frac{\sigma\left(g^{2}, s\right)}{g^{2}}\right) .
$$

We also measure the mass anomalous dimension $\gamma=d \ln m_{q} / d \ln \mu$ of the theory with 6 fermion flavours using the pseudoscalar density renormalization constant which is defined as

$$
Z_{P}(L)=\frac{\sqrt{3} f_{1}}{f_{P}(L / 2)}
$$

where

$$
\begin{aligned}
& f_{1}=\frac{-1}{12 L^{6}} \int d^{3} u d^{3} v d^{3} y d^{3} z\left\langle\bar{\zeta}^{\prime}(u) \gamma_{5} \lambda^{a} \zeta^{\prime}(v) \bar{\zeta}(y) \gamma_{5} \lambda^{a} \zeta(z)\right\rangle, \\
& f_{P}\left(x_{0}\right)=\frac{-1}{12 L^{6}} \int d^{3} y d^{3} z\left\langle\bar{\psi}\left(x_{0}\right) \gamma_{5} \lambda^{a} \psi\left(x_{0}\right) \bar{\zeta}(y) \gamma_{5} \lambda^{a} \zeta(z)\right\rangle,
\end{aligned}
$$

are correlation functions of the pseudoscalar density. Here sources $\zeta$ and $\zeta^{\prime}$ are located at the $t=0$ and $t=L$ boundaries, respectively. For these measurements the boundary matrices at $t=0$ and $t=L$ are set to unity. The mass step scaling function is then defined as:

$$
\begin{aligned}
& \Sigma_{P}(u, s, L / a)=\left.\frac{Z_{P}\left(g_{0}, s L / a\right)}{Z_{P}\left(g_{0}, L / a\right)}\right|_{g^{2}\left(g_{0}, L / a\right)=u}, \\
& \sigma_{P}(u, s)=\lim _{a / L \rightarrow 0} \Sigma_{P}(u, s, L / a),
\end{aligned}
$$

and we choose again $s=2$. We find the continuum step scaling function $\sigma_{P}$ by measuring $\Sigma_{P}$ at $L / a=6$ and 10 , and doing a quadratic extrapolation. It can be related to the anomalous dimension of the mass operator by

$$
\sigma_{P}(u, s)=\left(\frac{u}{\sigma(u, s)}\right)^{d_{0} /\left(2 b_{0}\right)} \exp \left[\int_{\sqrt{u}}^{\sqrt{\sigma(u, s)}} d x\left(\frac{\gamma(x)}{\beta(x)}-\frac{d_{0}}{b_{0} x}\right)\right],
$$

where $b_{0}=\beta_{0} /\left(16 \pi^{2}\right)$ in terms of the one-loop coefficient $\beta_{0}$ of the beta function and $d_{0}=$ $8 /\left(16 \pi^{2}\right)$ is the corresponding one-loop coefficient for the anomalous dimension, $\gamma=-d_{0} g^{2}$. This can be approximated at the fixed point with $\gamma^{*}\left(g^{2}\right)=-\frac{\sigma_{P}\left(g^{2}, s\right)}{\log (s)}$.

\section{Measurements and results}

The zero mass limit is determined by measuring the $\kappa_{c}=1 /\left(8+2 m_{0, c}\right)$ for all the used values of $\beta$ via the PCAC relation using lattice size $16^{4}$. The measured values of $\kappa_{c}$ are then used for all 

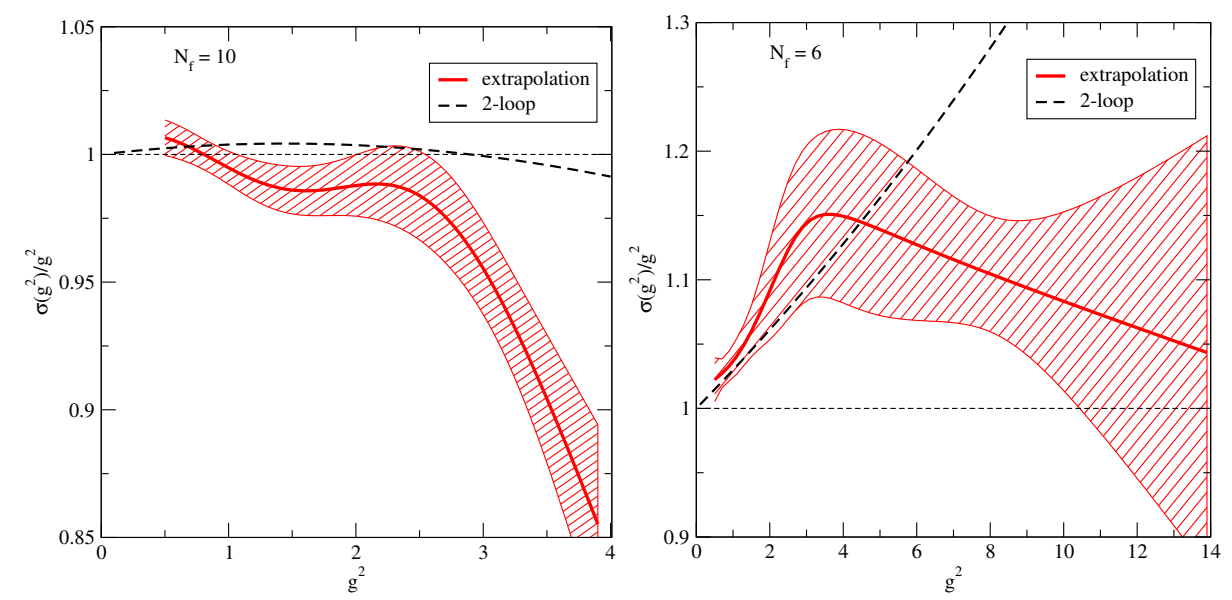

Figure 2: Step scaling function for $N_{f}=10$ (left) and $N_{f}=6$ (right). Continuous lines show 2-loop perturbative step scaling result.

lattice sizes. The running coupling $g$ is measured for $\beta=\{8,6,4,3,2,1.7,1.5,1.3,1\}$ for $N_{f}=10$ and $\beta=\{8,5,4,3,2.4,2,1.8,1.5,1.44,1.4,1.39\}$ for $N_{f}=6$, using lattice sizes $6^{4}, 8^{4}, 12^{4}$ and $16^{4}$. The measured values of $g$ are then used to find an interpolating function in $\beta$ of the form

$$
\frac{1}{g^{2}(\beta, L / a)}=\frac{\beta}{2 N} \sum_{i=0}^{n} c_{i}\left(\frac{2 N}{\beta}\right)^{i}
$$

with $c_{0}=1$. The interpolating function is used to find the step scaling function for $L / a=6,8$, and the continuum limit is extracted using

$$
\Sigma(u, 2, L / a)=\sigma(u, 2)+c(L / a)^{2} .
$$

Because of the improved action we expect the $\mathscr{O}(a)$ terms to be subleading. Unfortunately, with only two points in the extrapolation, it is not possible to verify the accuracy of the extrapolation quantitatively.

The anomalous dimension of the mass operator is determined similarly: The pseudoscalar density renormalization constant $Z_{P}$ is measured for $\beta=\{2.4,2,1.5,1.44,1.4,1.39\}$ and for $L / a=$ $6,8,10,12,16,20$, and these values are used to find an interpolating function of the form

$$
Z_{P}(\beta, L / a)=\sum_{i=0}^{n} c_{i}\left(\frac{1}{\beta}\right)^{i}
$$

with $c_{0}=1$. This is then used to calculate $\Sigma_{P}(u, 2, L / a)$, which is in turn extrapolated to the continuum limit with an order $(a / L)^{2}$ ansatz.

In figure 2 we show the step scaling functions. In the $N_{f}=10$ theory the evolution of the coupling is extremely slow, and our results basically agree with this at $g^{2} \lesssim 2.5$ : the step scaling practically vanishes in this range. In this case we expect the two-loop perturbative step scaling function to be fairly accurate, and from figure 2 we see that the errors should be an order of magnitude smaller in order to resolve it. At stronger coupling the measured step scaling deviates significantly from zero to negative values. Combined with the analytically known weak coupling behaviour, this 


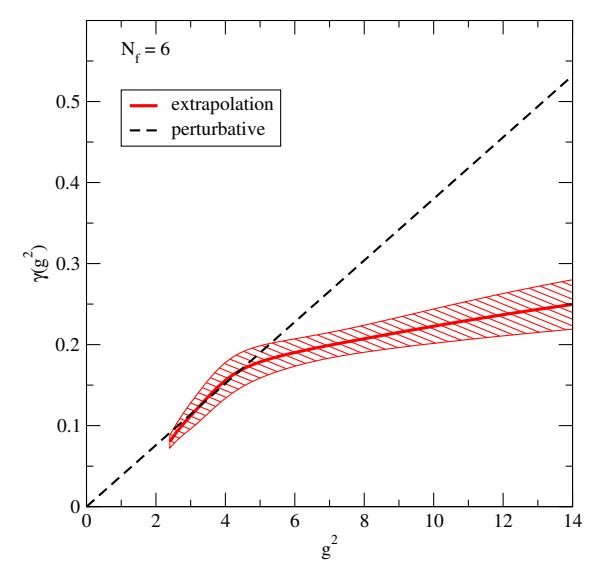

Figure 3: Anomalous dimension of the mass operator for $N_{f}=6$

indicates that the $\beta$-function must have a fixed point somewhere in this range. However, we believe that a large fraction of the observed deviation from the perturbative step scaling at strong coupling arises from the results at our strongest lattice coupling $\beta_{L}=4 / g_{0}^{2}=1$. This point deviates clearly from the rest of the simulation points, possibly indicating stronger cutoff effects.

In the $N_{f}=6$ theory the evolution of the coupling remains slow, which leads to large errors in the step scaling function. In this case we were able to reach mesured couplings up to $g^{2}=14$. However, the results indicate that the possible infrared fixed point is at $g^{2} \gtrsim 13$, and our statistical resolution is not sufficient to confirm or exclude the existance of an IRFP.

The measured values of mass anomalous exponent $\gamma$ are shown in figure 3. It shows that $0.1<\gamma<0.3$. Because the value of the anomalous dimension of the mass operator is only scheme independent at the fixed point, these results are not that interesting, since we were unable to find the IRFP.

\section{Conclusions}

Our simulations verify that the SU(2) gauge theory with 6 flavours of fundamental representation fermions is indeed close to the lower edge of the conformal window. Unfortunately, the possible fixed point in this theory is at such a strong coupling that we were not able to fully resolve the behaviour: the results are compatible either with a fixed point at $g^{2} \gtrsim 12$ or with a "walking" behaviour where the $\beta$-function almost vanishes. The value of the fixed point coupling is naturally scheme dependent; this value is for Schrödinger functional scheme. To resolve this question requires simulations with an action which can be used at stronger lattice couplings than used in this work.

\section{Acknowledgments}

T.K. is supported by the Magnus Ehrnrooth foundation and by University of Jyväskylä Faculty of Mathematics and Science. J.R. is supported by the Finnish Academy of Science and Letters Väisälä fund. We acknowledge the support from the Academy of Finland grant number 1134018. The computations have been performed at the Finnish IT Center for Science. 


\section{References}

[1] H. Georgi, Phys. Rev. Lett. 98, 221601 (2007)

[2] S. Weinberg, Implications Of Dynamical Symmetry Breaking: An Addendum, Phys. Rev. D 19, 1277 (1979); L. Susskind, Dynamics Of Spontaneous Symmetry Breaking In The Weinberg-Salam Theory, Phys. Rev. D 20, 2619 (1979).

[3] C. T. Hill and E. H. Simmons, Phys. Rept. 381, 235 (2003) [Erratum-ibid. 390, 553 (2004)] [arXiv:hep-ph/0203079].

[4] F. Sannino, Dynamical Stabilization of the Fermi Scale: Phase Diagram of Strongly Coupled Theories for (Minimal) Walking Technicolor and Unparticles, arXiv:0804.0182 [hep-ph].

[5] F. Bursa, L. Del Debbio, L. Keegan, C. Pica and T. Pickup, Mass anomalous dimension and running of the coupling in SU(2) with six fundamental fermions, arXiv:1010.0901 [hep-ph].

[6] S. Catterall and F. Sannino, Minimal walking on the lattice, Phys. Rev. D 76, 034504 (2007) [arXiv:0705.1664 [hep-lat]].

[7] A. J. Hietanen, J. Rantaharju, K. Rummukainen and K. Tuominen, Spectrum of SU(2) lattice gauge theory with two adjoint Dirac flavors, JHEP 0905, 025 (2009) [arXiv:0812.1467 [hep-lat]]

[8] L. Del Debbio, A. Patella and C. Pica, Higher representations on the lattice: numerical simulations. SU(2) with adjoint fermions, Phys. Rev. D 81 (2010) 094503 [arXiv:0805.2058 [hep-lat]].

[9] S. Catterall, J. Giedt, F. Sannino and J. Schneible, Phase diagram of SU(2) with 2 flavors of dynamical adjoint quarks, arXiv:0807.0792 [hep-lat].

[10] A. J. Hietanen, K. Rummukainen and K. Tuominen, Evolution of the coupling constant in SU(2) lattice gauge theory with two adjoint fermions, Phys. Rev. D 80, 094504 (2009) [arXiv:0904.0864 [hep-lat]].

[11] T. DeGrand, Y. Shamir, B. Svetitsky, Infrared fixed point in SU(2) gauge theory with adjoint fermions, [arXiv:1102.2843 [hep-lat]].

[12] T. Appelquist, G. T. Fleming and E. T. Neil, Lattice Study of the Conformal Window in QCD-like Theories, Phys. Rev. Lett. 100, 171607 (2008) [arXiv:0712.0609 [hep-ph]].

[13] Z. Fodor, K. Holland, J. Kuti, D. Nogradi and C. Schroeder, Nearly conformal gauge theories in finite volume, Phys. Lett. B 681, 353 (2009) [arXiv:0907.4562 [hep-lat]];

[14] A. Deuzeman, M. P. Lombardo and E. Pallante, The physics of eight flavours, Phys. Lett. B 670, 41 (2008) [arXiv:0804.2905 [hep-lat]];

[15] Y. Shamir, B. Svetitsky and T. DeGrand, Zero of the discrete beta function in SU(3) lattice gauge theory with color sextet fermions, Phys. Rev. D 78, 031502 (2008) [arXiv:0803.1707 [hep-lat]].

[16] B. Sheikholeslami and R. Wohlert, Nucl. Phys. B 259, 572 (1985).

[17] M. Luscher, R. Narayanan, P. Weisz and U. Wolff, Nucl. Phys. B 384, 168 (1992) [arXiv:hep-lat/9207009].

[18] S. Sint and R. Sommer, Nucl. Phys. B 465, 71 (1996) [arXiv:hep-lat/9508012].

[19] R. Sommer, Nucl. Phys. Proc. Suppl. 60A, 279 (1998) [arXiv:hep-lat/9705026].

[20] M. Luscher and P. Weisz, Nucl. Phys. B 479, 429 (1996) [arXiv:hep-lat/9606016].

[21] T. Karavirta, A. Mykkanen, J. Rantaharju, K. Rummukainen, K. Tuominen, Nonperturbative improvement of SU(2) lattice gauge theory with adjoint or fundamental flavors, [arXiv:1101.0154 [hep-lat]]. 